\title{
THE EXISTENCE OF DEUTEROTOKOUS REPRODUCTION MODE IN THE T. TABACI (THYSANOPTERA: THRIPIDAE) CRYPTIC SPECIES COMPLEX
}

\author{
Wondimagegn Atilaw WOLDEMELAK* \\ Szent István University, Department of Entomology, Villányi str. 29-43, Budapest, Hungary
}

Received: December 2019; Accepted: May 2020

\begin{abstract}
The present study aimed to investigate whether adult males could fertilize immature female pupae in the leek (L1) and tobacco-associated (T) Thrips tabaci lineages and to investigate the potential effects of mother to son inbreeding on the appearance of deuterotokous females in the T lineage. To confirm the pupal insemination, a single female pupa was exposed to a single adult male for 24 hours. The removal of a male from the tube that contained the female pupa was made before the female pupa emerged to adulthood. Emerged female adults were isolated and reared individually and the sex of their progeny was determined in the larval stages. To examine a mother and son inbreeding, a single female adult was exposed to her son for 48 hours, and for two consecutive generations, the mode of reproduction was determined by identifying the sex of the progeny. This result showed that pupal insemination was not successful; thus, all the tested females produced only male progeny. Virgin inbred females produced both male and female progeny that refers to deuterotokous reproduction mode. Three virgin females produced a total of 20 females and 75 males in two consecutive generations. A study concluded that deuterotokous mode of reproduction is not a persistent mode of reproduction in the $\mathrm{T}$ lineage but is a result of irregularity in the mode of reproduction, and it might be changed because of brother and sister inbreeding and need further studies.
\end{abstract}

Key word: arrhenotoky, mother to son inbreeding, pupal insemination, thelytoky, virgin

\section{INTRODUCTION}

Thysanoptera insect species were classified under the hemimetabola postembryonic development, because they do not follow distinctive hemimetabolous developmental stages. They convey the transition between hemimetabolous and holometabolous postembryonic stages because the comparison of an immature nymph with adult exhibit many similarities (Moritz 1995).

The life cycle of Thrips tabaci Lindeman (Thysanoptera: Thripidae) includes an egg, first and second instar larvae, pre-pupa, pupa, and adult (Lewis 1973), and all these stages pass through moulting. After hatching, the first and second instar larvae start to feed. Pre-pupa and pupa stages are inactive and non-feeding. Pre-pupae and pupae are found in the soil, at the base of the onion plant neck, or underneath bulb scales to protect themselves against environmental effects.
The reproductive organ of the pupa develops prematurely; for this reason, immediate egg fertilization is not common. However, a most unusual form of coupling has been reported in Limothrips denticornis Haliday in which a mature male mated with female pre-pupa (Lewis 1973). The adult males of Frankliniella occidentalis Pergande were able to mate with the last stages of female (Ogada et al. 2015 not published). Insect's reproductive organs comprise a spermatheca that are used to store male sperm during hibernation and to fertilize the eggs laid next spring (Lewis 1973).

On the basis of the mitochondrial DNA data, reproduction mode, and host adaptation, T. tabaci has three major evolutionary lineages; two of them are associated with leek (L1 and L2), whereas the third one is associated with tobacco $(\mathrm{T})$ (Brunner et al. 2004). 
The most common modes of reproduction in T. tabaci lineages are thelytoky (females produced from unfertilized eggs), arrhenotoky (males produced from unfertilized eggs and females produced from fertilized eggs), and uncommonly deuterotoky - females and males produced from unfertilized eggs (Fekrat et al. 2014; Li et al. 2014; Morsello et al. 2008; Nault et al. 2006; Sogo et al. 2015). L1 and T types are following arrhenotokous reproductive mode, and L2 type is following thelytokous reproduction mode. The deuterotoky reproduction mode is not well documented for the whole Thysanoptera insect species, except for T. tabaci (Nault et al. 2006) and Apterothrips apteris (Daniel) (Strauss \& Karban 1994). There are some suspected reasons that such mode of reproduction is due to some external factors such as pupal insemination. In some cases, although the adult male mated with the immature female, the male sperm might be stored in the immature female spermatheca. Thus, later on, when this female became an adult, this male sperm could be used to fertilize the eggs, and thus, an already mated female during the immature stage will be producing a combination of male and female progenies. The second factor might include the mating between mother and son resulting in inbreeding depression that caused reproductive irregularity by increasing the homogeneity and decreasing heterogeneity of individual genome. Such external factors might lead to wrong conclusions regarding deuterotokous mode of reproduction.

The aim of this study was to reveal if adult male could fertilize immature female pupa in the arrhenotokous L1 and T types and to identify if the mother to son inbreeding could lead to the existence of deuterotokous mode of reproduction in the arrhenotokous $\mathrm{T}$ type.

\section{MATERIAL AND METHODS}

\section{Pupal insemination \\ Rearing}

To begin the experiment, 12 adult females with the unknown age was isolated from the culture of each lineage (12 females from L1 and 12 females from T) type. They were reared individually in 2-ml micro-centrifuge tubes with single leaf discs of their ideal host plants (cabbage leaf discs for L1 and tobacco leaf discs were provided for $\mathrm{T}$ ) as a food and oviposition substrate. The leaf discs were replaced every 24 hours. The rearing has been carried out under the environmental growth chamber at $23{ }^{\circ} \mathrm{C}$ with $16: 8$ (light : dark). The eggs in the leaf discs have checked with an under-light stereomicroscope. The dead mothers were preserved in $96 \%$ alcohol and their mitochondrial cytochrome c oxidase subunit I (MT-COI) gene was sequenced to confirm the lineage (Farkas et al. 2020). The hatched first and second instar larvae were individually transferred to new tubes with fresh leaf discs and raised to the pupal stage. To test the pupal insemination, 42 female pupae from L1 and 44 female pupae from $T$ type were used. In order to investigate the response of immature pupa upon mating with an adult male, a single female pupa was confined to a single adult male in a $2-\mathrm{ml}$ microcentrifuge tube for 24 hours. The response of female pupa to the adult male during mating was checked using a stereomicroscope.

To avoid the mating between newly emerged female adult with male adult, a male from the tube that contained the female pupa was removed before the newly female adult emerge and the female pupa was left on the leaf discs until they became an adulthood. The newly emerged female adults were reared for 8-10 days to produce eggs and all their newly hatched first and second instar larvae were collected and preserved in $75-80 \%$ ethanol alcohol. This immature preservation method was suggested by Morison (1947). After that, the preserved first and second instars of L1 and T types were slide mounted to identify their sex.

At a later period, 439 first and 219 second instars of $\mathrm{L} 1$ and $\mathrm{T}$ lineages were mounted by pouring few drops of Berlese mount medium on the microscopic flattened rectangular glass. After adding the larvae specimens to the drop of Berlese mounting medium, the microscopic glass was covered with a glass coverslip. To dry the specimens, the slides were kept in an oven at $50{ }^{\circ} \mathrm{C}$ for 2 days.

\section{Identification of larva stages}

For immature identification of the first and second instar larva, the technique described by Speyer and Parr (1941) was used. This identification key for Thripidae is based on the number of setae 
on the II to VII abdominal segments and the number of setae on their pronotum, and the differences between first and second instar larvae are listed below.

1. First instar larvae is small in size when compared with the second instar larvae, and it comprises two pairs of sternal setae on the abdominal segments II to VII and six pairs of setae on the pronotum (Vierbergen et al. 2010). It also contains one pair of spiracle on the abdominal segment II (Kirk 1987).

2. Second instar larvae has a larger and fat body when compared with first instar larvae. This stage consists of three pairs of sternal setae on the abdominal segments II to VII, two pairs of spiracles, and seven pairs of setae on the pronotum (Vierbergen et al. 2010). It has no spiracle on the abdominal segment II (Kirk 1987).

\section{Sex identification}

The sex of the first and second instar larvae in the family Thripidae is determined based on their abdominal segment IX. The first instar male larvae exhibit four pairs of setae and female larvae exhibit three pairs of setae on their abdominal segment IX, whereas the second instar male larvae exhibit five pairs of setae and female larvae exhibit four pairs of setae on their abdominal segment IX (Vierbergen et al. 2010). All the features and sex identification of the first and second instar larvae were examined using Leica DMLB binocular light microscope (Leica Microsystem GmbH, Wetzlar, Germany) with $400 \times, 600 \times$ and $1000 \times$ magnifications.

\section{Checking of insemination}

To determine the female pupal insemination, the sex of the progeny was identified at first and second instar pre-pupa, pupa, and adult stages. Thus, the female pupa was considered as inseminated by adult male if there is a single female from her progenies, and the female pupa was considered as not inseminated by adult male if all the progenies are males.

\section{Reproduction mode in $T$ type $T$. tabaci lineage}

$\mathrm{T}$ type lineage is reproducing through the arrhenotokous (sexual) mode of reproduction in which the virgin females produce only males and the mated females produce both males and females. The sex identification of adult progeny was the method used to determine the mode of reproduction in $\mathrm{T}$ type.
A virgin female producing only male progeny was considered as arrhenotoky female, and a virgin female producing a combination of male and female progeny was considered as a deuterotoky female. To confirm the arrhenotokous and deuterotokous modes of reproduction in the tested individuals, the following procedure was used: 20 female adults of unknown age were isolated randomly from $\mathrm{T}$ type culture and reared separately in 2-ml micro-centrifuge tubes. The tobacco leaf discs were used to serving as a food source and oviposition site. Before the tobacco leaf discs were provided as a food and oviposition object, all tobacco leaves were checked under a light microscope to ensure that the leaf discs were not contaminated by other thrips. The progeny from these mothers were raised to adulthood also in isolation after egg hatching and were used as $\mathrm{F}_{0}$ generation. The reason to use these progenies as $\mathrm{F}_{0}$ generation is that their mothers were taken from the culture and had been exposed to high densities of males and experienced to mate by any other males and it makes difficult to determine whether the females were produced either through the arrhenotokous or deuterotokous mode of reproduction.

\section{Mother and son inbreeding}

To test whether a mother and son inbreeding is the factor to induce deuterotokous mode of reproduction in $\mathrm{T}$ lineage, 10 females of unknown age were isolated randomly from stock culture and reared separately in 2-ml micro-centrifuge tubes. The tobacco leaf discs were free of contamination by other thrips species. The newly hatched progeny from these mothers were raised to adulthood. Those progenies were considered as $F_{1}$ generation and used as a parental generation for the subsequent inbred line. Thus, all the tested inbred generations were assumed to be related. Mother and son inbreeding has tested for two subsequent generations such as $\left(\mathrm{F}_{1}\right)$ females were daughters of females taken from stock culture and used as a parental generation in the experiment and $\left(F_{2}\right)$ females were daughters of $F_{1}$ inbred females.

To ensure a possibility to obtain a son from virgin female, 30 virgin female adults of $\mathrm{T}$ lineage were isolated from the $F_{1}$ females and reared separately on the tobacco leaf discs for 2 days at $23{ }^{\circ} \mathrm{C}$. 
As they were virgins, their eggs would not be fertilized and only sons would arise. Then the reproducing females were placed at $15^{\circ} \mathrm{C}$. At this low temperature, the mother's longevity has increased, which increases the chance for mating with their sons. However, some mothers died within a relatively short period before mating with their sons. They were excluded from this test. When the son became adult, single mother and son were confined into the same micro-centrifuge tube for $48 \mathrm{~h}$. Then the male was removed, and its mother was kept isolated individually for the rest of her life. The same procedure was used for $\mathrm{F}_{2}$ mothers and sons inbreeding test. The relatedness of isolated individuals in the $F_{1}$ and $F_{2}$ progenies were recorded, and $F_{2}$ sisters were produced by the same $F_{1}$ inbred female (Fig. 1).
MT-COI genes and sex determination at the adult and nymphal stages were the criteria applied to describe the lineage and mode of reproduction, respectively. A virgin inbred female produced a combination of male and female was considered as a deuterotokous female.

\section{RESULTS}

\section{Morphological description of the first and sec- ond instar larvae of $T$. tabaci}

The morphological identifications of these two lineages larvae were based on the number of setae on their pronotum and the number of setae on the abdominal segments II to III. As described in the methodology, first instar larvae consisted of one pair of setae on the ventral abdominal segments II to III and six pairs of setae on the pronotum (Figs. 2 A, B and 3 A, B).
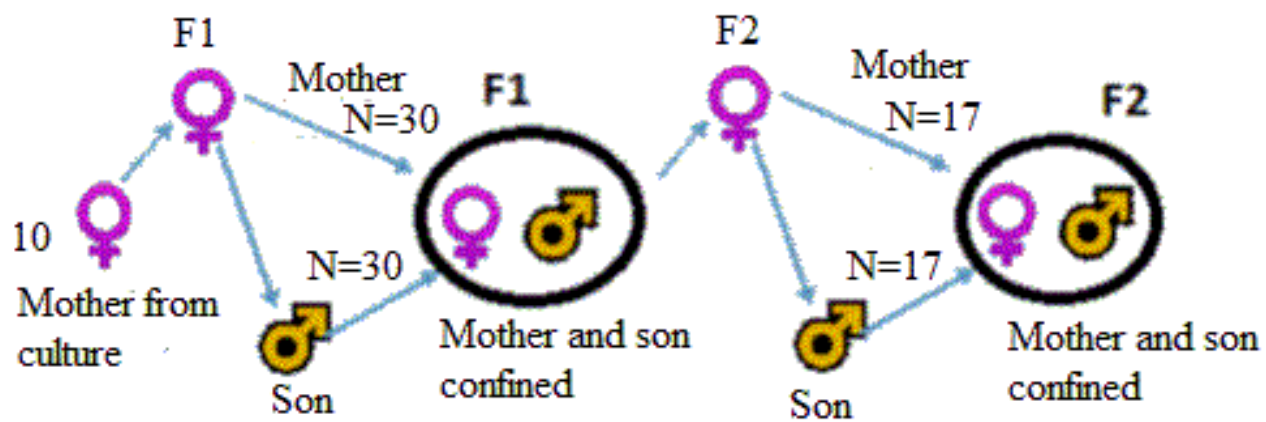

Fig. 1. Mother and son inbreeding scheme in T lineage: 10 females were taken from the stock culture and reared them to produce F1 progeny. Then, $30 \mathrm{~F} 1$ virgin females were reared to produce son and when the son became an adult; both mother and son were confined in the same tube for $48 \mathrm{~h}$. The male was removed from the tube after $48 \mathrm{~h}$ and its mother kept reared on tobacco leaf discs and named as the F1 generation. Since then, F1 female allowed to produce a son to continue the F2 mother and son inbreeding and similar procedures were used with F1 inbreeding line
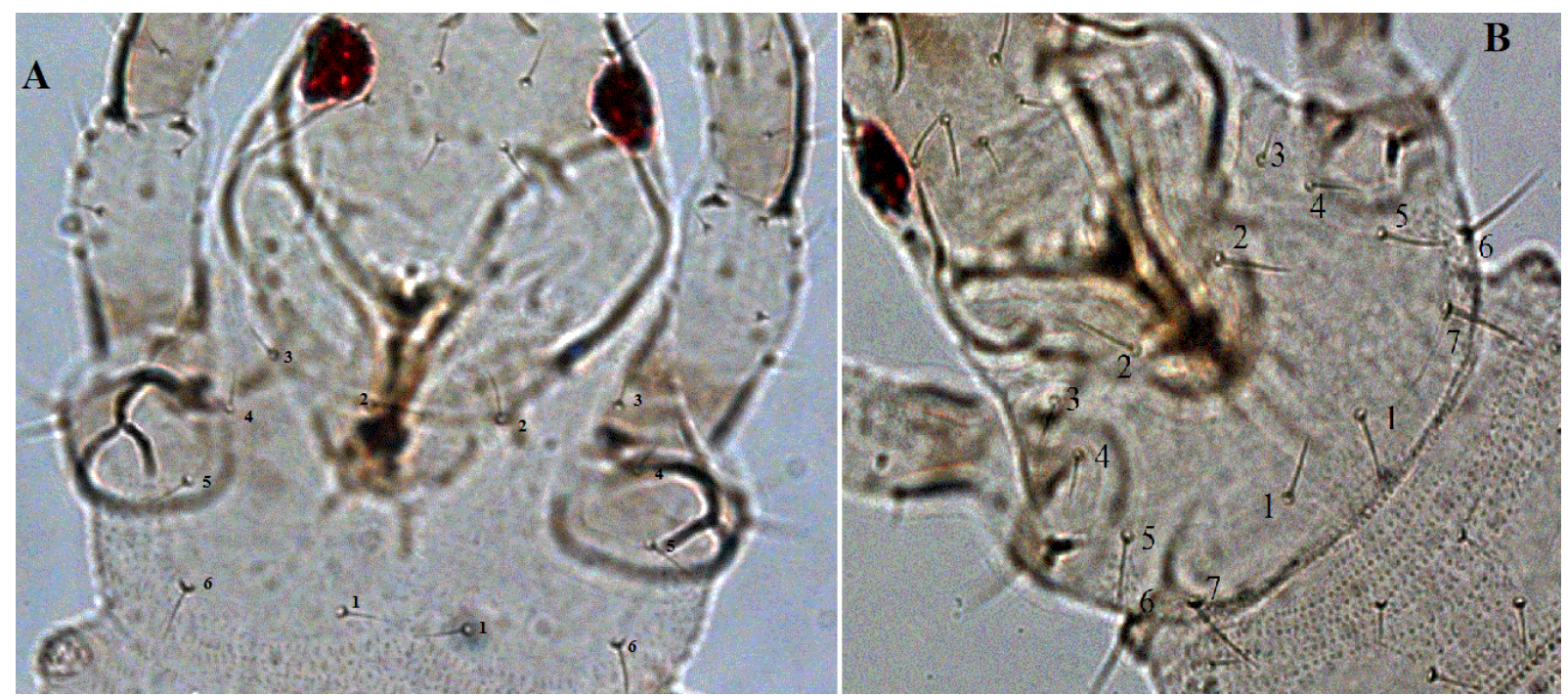

Fig. 2. (A) Indicated that the first instar larvae and the numbers are used to indicate the pairs of setae on the pronotum; (B) Indicated that the second instar larvae and the numbers are used to indicate the pair of setae on the pronotum 

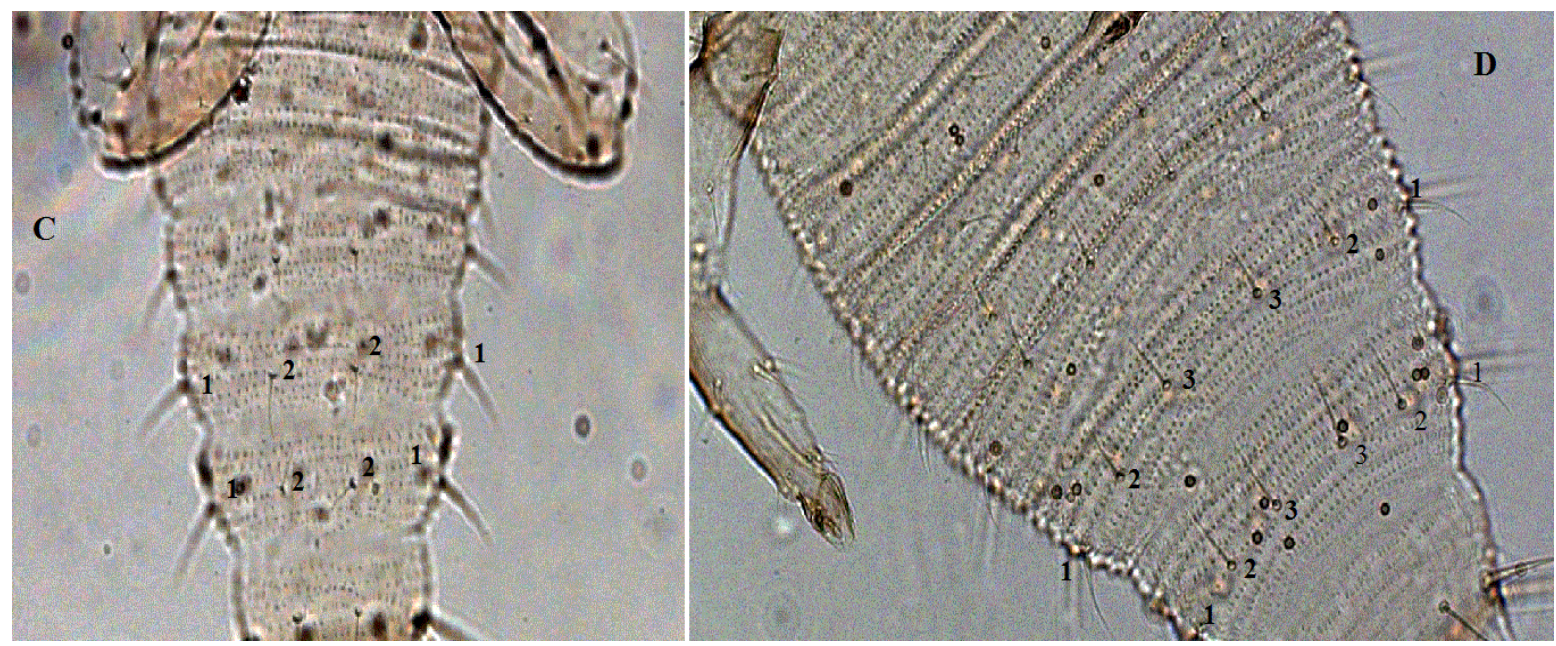

Fig. 3. (C) Indicated that the first instar larvae and the numbers are used to indicate the pairs of setae on the ventral part of the abdomen; (D) Indicated that the second instar larvae and the numbers are used to indicate the pair of setae on the ventral part of the abdomen

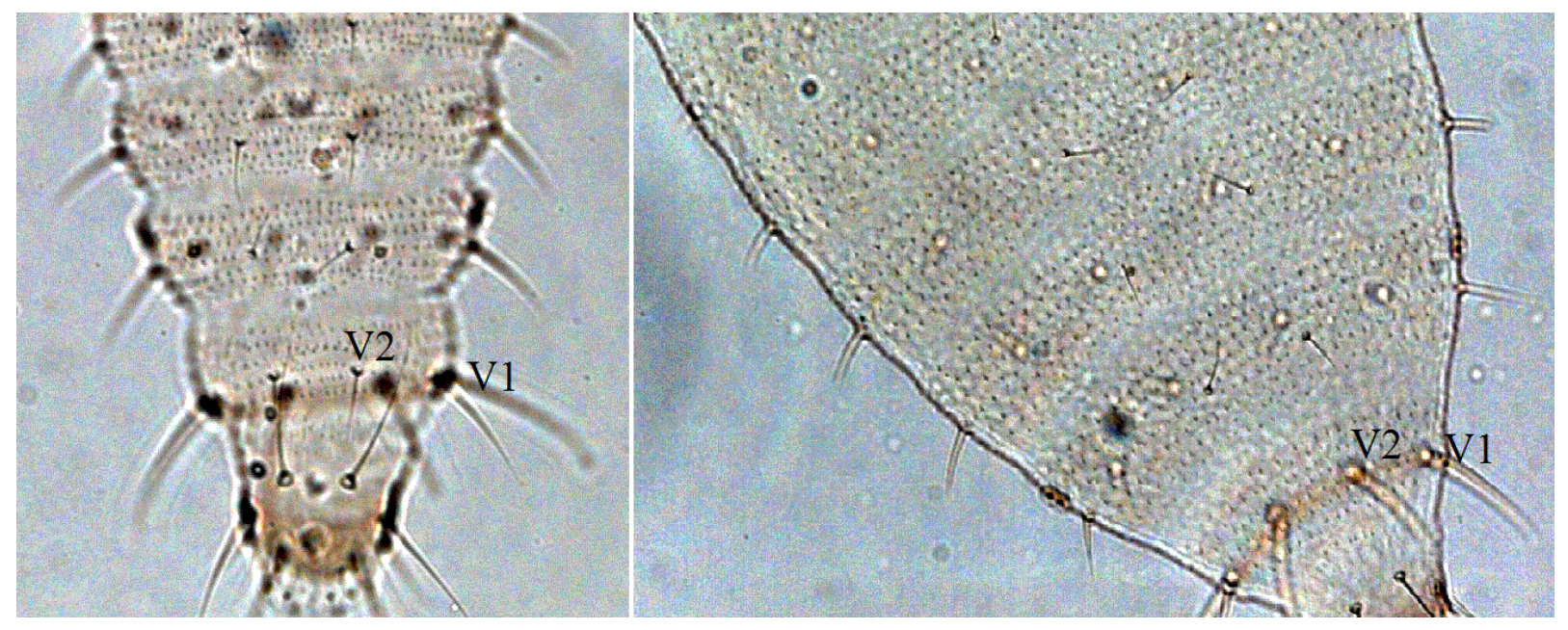

Fig. 4. First instar male. The numbers are used to indicate the pairs of setae on both dorsal and ventral abdominal segment IX
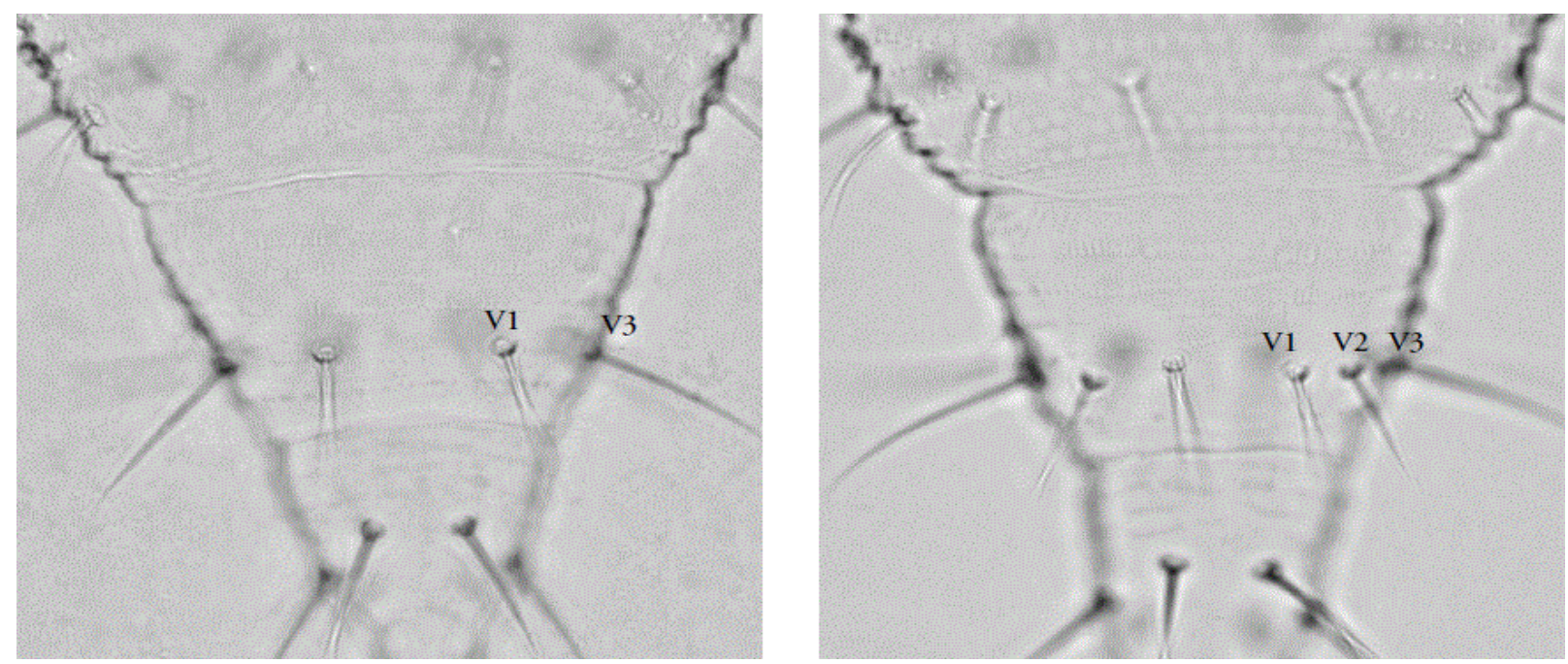

Fig. 5. Second instar male. The numbers are used to indicate the pairs of setae on both dorsal and ventral abdominal segment IX 


\section{Sex identification keys}

On the basis of the morphological identifications of first and second instar larvae, the sex of the immature progeny was determined based on their number of setae on the abdominal segment of IX. First instar male larvae consisted of four pairs of setae on both dorsal and ventral of abdominal segment IX (Fig. 4) and second instar male larvae consisted of five pairs of setae on both dorsal and ventral abdominal segment IX (Fig. 5).

\section{Pupal insemination}

According to this experiment result, pupal insemination is not possible in the T. tabaci lineages within 24 hours of mating. Thus, all the tested females produced only male progeny (Table 1).

Table 1. The sex of progeny produced from virgin $\mathrm{L} 1$ and $\mathrm{T}$ type females that were exposed to male adult at pupal stage

\begin{tabular}{cccccc}
\hline Lineages & $\begin{array}{c}\text { First } \\
\text { instar } \\
\text { male }\end{array}$ & $\begin{array}{c}\text { Second } \\
\text { instar } \\
\text { male }\end{array}$ & $\begin{array}{c}\text { Pupae } \\
\text { progeny }\end{array}$ & $\begin{array}{c}\text { Adult } \\
\text { male } \\
\text { progeny }\end{array}$ & $\begin{array}{c}\text { Total } \\
\text { male }\end{array}$ \\
\hline L1 & 187 & 95 & 66 & 118 & 466 \\
\hline $\mathrm{T}$ & 252 & 121 & 22 & 127 & 522 \\
\hline
\end{tabular}

\section{Mother to son inbreeding}

Deuterotokous reproduction mode has encountered during mother and son inbreeding test. At $\mathrm{F}_{2}$ generation, the inbred virgin female taken from $\mathrm{F}_{1}$ generation produced a combination of male and female progenies, and this female was considered as a deuterotoky ones (Table 2).

Table 2. The existence of deuterotokous mode of reproduction from $\mathrm{F}_{2}$ mother and son inbreeding

\begin{tabular}{cccccc}
\hline $\begin{array}{c}\text { Genera- } \\
\text { tion }\end{array}$ & $\begin{array}{c}\text { No. of } \\
\text { inbred } \\
\text { female }\end{array}$ & & \multicolumn{3}{c}{ Male Female } \\
mode & & $\begin{array}{c}\text { Reproduction Deuterotoky } \\
\text { mother }\end{array}$ \\
\hline$F_{1}$ & 30 & $\checkmark$ & & Arrhenotoky & 0 \\
\hline$F_{2}$ & 17 & $\checkmark$ & $\checkmark$ & Deuterotoky & $1 / 17$ \\
\hline
\end{tabular}

The deuterotoky females produced from inbred females for three consecutive generations. About 20 females and 75 males were produced for two consecutive generations. $F_{1 D}$ female has produced 10 deuterotoky females, and 2 of them died at the pupal stage; of the 8 deuterotoky females, only 1 was continued to produce both males and females and the rest 7 females were turned to their original arrhenotokous mode of reproduction. Similarly, at $\mathrm{F}_{2 \mathrm{D}}$ generation, of the eight deuterotoky females, only one virgin female yielded both male and female progenies and the rest seven females were turned to their original arrhenotokous reproduction mode. After three generations, all the deuterotoky females turned to their original arrhenotokous mode of reproduction (Table 3 ).

Table 3. A sex ratio of progeny that were produced by deuterotoky virgin female for three consecutive generation

\begin{tabular}{|c|c|c|c|c|}
\hline \multirow{2}{*}{ Generation } & \multirow{2}{*}{$\begin{array}{c}\text { No. of deu- } \\
\text { terotoky } \\
\text { female }\end{array}$} & \multicolumn{2}{|c|}{$\begin{array}{l}\text { No. of progeny from } \\
\text { deuterotoky female }\end{array}$} & \multirow{2}{*}{$\begin{array}{l}\text { Reproductive } \\
\text { mode }\end{array}$} \\
\hline & & Male & Female & \\
\hline $\mathrm{F}_{0 \mathrm{D}}{ }^{\mathrm{a}}$ & 1 & 5 & 8 & Deuterotokous \\
\hline $\mathrm{F}_{1 \mathrm{D}}{ }^{\mathrm{b}}$ & $1 / 8$ & 4 & 10 & Deuterotokous \\
\hline $\mathrm{F}_{2 \mathrm{D}^{\mathrm{c}}}$ & $1 / 8$ & 66 & 2 & Deuterotokous \\
\hline $\mathrm{F}_{3 \mathrm{D}}{ }^{\mathrm{d}}$ & 2 & 72 & 0 & Arrhenotokous \\
\hline
\end{tabular}

${ }^{\mathrm{a}} \mathrm{F}_{0 \mathrm{D}}$ - the deuterotoky female produced from $\mathrm{F}_{2}$ inbred mother; ${ }^{b} F_{1 D}$ - the deuterotoky female progeny produced from $F_{0 D}$ female; ${ }^{c} F_{2 D}$ - the deuterotoky female progeny produced from $F_{1 D}$ female; ${ }^{\mathrm{d}} \mathrm{F}_{3 \mathrm{D}}$, the female produced from $\mathrm{F}_{2 \mathrm{D}}$ female

\section{DISCUSSION AND CONCLUSION}

The genetic variability of onion thrips has confirmed that $T$. tabaci is not a single pest species but rather a cryptic species complex, which is based on significant differences between the lineages regarding the mode of reproduction and host plant preferences, and that there is considerable genetic variability within the three lineages. The currently recognized lineages are arrhenotokous leek (L1), thelytokous leek (L2), and arrhenotokous tobaccoassociated (T) $T$. tabaci lineages.

All modes of reproduction have been reported in T. tabaci populations. However, deuterotoky is relatively uncommon mode of reproduction in $T$. tabaci because it has been reported only by Nault et al. (2006). It is suspected that such mode of reproduction is due to some other ways. The first way might be pupal insemination by adult males. Adult males and female pupae coexist together in the field, and insemination or mating between them could be expected. 
Insemination at pupal stage upon the metamorphosis to adult stage is possible because of the storage of the male sperm in pupae spermatheca and using this sperm to fertilize eggs. Therefore, mixed progeny of male and female offspring are produced by the mated arrhenotokous female. This phenomenon was reported in $L$. denticornis in which a mature male was found to mate with a female pre-pupa (Lewis 1973). The second factor is the mating between mother and son resulting in inbreeding depression that caused reproductive irregularity.

The determination of mode of reproduction in T. tabaci lineage based on the sex of progeny takes more time. A time-saving technique for differentiation of the mode of reproduction using the COI sequences was reported by Toda and Murai (2007). However, sometimes the lineages showing arrhenotoky were reported wrongly as showing thelytoky according to the COI-based technique (Aizawa et al. 2016). This might be due to the interbreeding between arrhenotokous and thelytokous T. tabaci lineages, resulting in the thelytoky female carrying arrhenotoky male-originated nuclear gene (Li et al. 2015). Heteroplasmy, where females carry multiple mitochondrial DNA genes within the cell, has been reported in T. tabaci population (Gawande et al. 2017).

This result has shown that adult males were attempting to mate with the female pupae. However, the mating was not successful; consequently, only male progeny was produced by all the tested progenies $(\mathrm{N}=988)$ in the arrhenotokous leek (L1) and tobacco-associated (T) T. tabaci lineages. There are feasible reasons to explain the failure of female pupa to inseminate by an adult male, such as (1) this stage is inactive and could not identify the sex hormone produced by the male to mate; (2) the developmental stages of $T$. tabaci are passed through molting and adult male genital might not get the female pupa genital part because the molt skin might cover the female pupa reproductive part; (3) the pupal stage of T. tabaci is inactive for feeding and, at the same time, the reproductive part might also not well developed.

Since Nault et al. (2006) report, deuterotokous mode of reproduction has never been reported again in T. tabaci, and it still might be thought of as mere developmental irregularities. Such a scenario led to do this research, and pupal insemination was the suspected factor.
However, this result has shown that insemination at pupal stage is not possible in $T$. tabaci lineages. The other possibility might be mating between relatives, which led to induce depression effects on the next generation, and this result has shown that mother and son inbreeding has induced deuterotokous mode of reproduction in the $\mathrm{T}$ lineage (the virgin inbred female produced the combination of male and female progenies). Therefore, the mating between mother and son potentially change the arrhenotoky female to deuterotokous mode of reproduction in the $\mathrm{T}$ lineage. The deuterotokous females in the $\mathrm{T}$ lineage were reared for three subsequent generations, and their progeny sex ratio was female biased at $F_{0 D}$ and $F_{1 D}$ generations (Table 3), and during the $F_{2 D}$ generation, the proportion of males were higher than females. Lastly, the deuterotokous females turned back to their original arrhenotokous mode of reproduction. $\mathrm{F}_{3 \mathrm{D}}$ deuterotokous females produced all male progeny. Therefore, this result indicated that deuterotokous mode of reproduction is not a persistent mode of reproduction in the $\mathrm{T}$ type but is a result of irregularity in the mode of reproduction. The variation in the mode of reproduction and genetic diversity among T. tabaci lineages changes across time even though when they shared the same COI haplotype (Nault et al. 2006; Jacobson et al. 2016). Inbreeding between brother and sister might induced the deuterotokous mode of reproduction, and it must be examined in future studies.

\section{REFERENCES}

Aizawa M., Watanabe T., Kumano A., Miyatake T., Sonoda S. 2016. Cypermethrin resistance and reproductive types in onion thrips, Thrips tabaci (Thysanoptera: Thripidae). Journal of Pesticide Science 41: 167-170. DOI: 10.1584/jpestics.d16-049.

Brunner P.C., Chatzivassiliou E.K., Katis N.I., Frey J.E. 2004. Host-associated genetic differentiation in Thrips tabaci (Insecta; Thysanoptera), as determined from mtDNA sequence data. Heredity 93: 364-370. DOI: 10.1038/sj.hdy.6800512.

Farkas P., György Z., Tóth A., Sojnóczki A., Fail J. 2020. A simple molecular identification method of the Thrips tabaci (Thysanoptera: Thripidae) cryptic species complex. Bulletin of Entomological Research 110(3): 397-405. DOI: $10.1017 / \mathrm{s} 0007485319000762$. 
Fekrat L., Manzari S., Shishehbor P. 2014. Morphometric and molecular variation in Thrips tabaci Lindeman (Thysanoptera: Thripidae) populations on onion and tobacco in Iran. Journal of Agricultural Science and Technology 16: 1505-1516.

Gawande S.J., Anandhan S., Ingle A.A., Jacobson A., Asokan R. 2017. Heteroplasmy due to coexistence of mtCOI haplotypes from different lineages of the Thrips tabaci cryptic species group. Bulletin of Entomological Research 107: 534-542. DOI: 10.1017/s0007485317000025.

Jacobson A.L., Nault B.A., Vargo E.L., Kennedy G.G. 2016. Restricted gene flow among lineages of Thrips tabaci supports genetic divergence among cryptic species groups. PLoS ONE 11; e0163882; 16 p. DOI: 10.1371/journal.pone.0163882.

Kirk W.D.J. 1987. A key to the larvae of some common Australian flower thrips (Insecta, Thysanoptera), with a host-plant survey. Australian Journal of Zoology 35: 173-185. DOI: 10.1071/zo9870173.

Lewis T. 1973. Thrips. Their biology, ecology and economic importance. Academic Press, UK, 350 p.

Li X.W., Fail J., Wang P., Feng J.N., Shelton A.M. 2014. Performance of arrhenotokous and thelytokous Thrips tabaci (Thysanoptera: Thripidae) on onion and cabbage and its implications on evolution and pest management. Journal of Economic Entomology 107: 1526-1534. DOI: 10.1603/ec14070.

Li X.W., Wang P., Fail J., Shelton A.M. 2015. Detection of gene flow from sexual to asexual lineages in Thrips tabaci (Thysanoptera: Thripidae). PLoS ONE 10; e0138353; 13 p. DOI: 10.1371/journal.pone.0138353.

Morison G.D. 1947. Thysanoptera of the London area. Part I. London Naturalist 26 (Supplement): 1-36.

Moritz G. 1995. Morphogenetic development of some species of the order Thysanoptera (Insecta). In: Parker B.L., Skinner M., Lewis T. (Eds.), Thrips Biology and Management. Series A: Life Sciences 276: 489-504. DOI: 10.1007/978-1-4899-1409-5_78.

Morsello S.C., Groves R.L., Nault B.A., Kennedy G.G. 2014. Temperature and precipitation affect sea- sonal patterns of dispersing tobacco thrips, Frankliniella fusca, and onion thrips, Thrips tabaci (Thysanoptera: Thripidae) caught on sticky traps. Environmental Entomology 37(1): 79-86. DOI: 10.1603/0046-225x(2008)37[79:tapasp]2.0.co;2.

Nault B.A., Shelton A.M., Gangloff-Kaufmann J.L., Clark M.E., Werren J.L., Cabrera-La Rosa J.C., Kennedy G.G. 2006. Reproductive modes in onion thrips (Thysanoptera: Thripidae) populations from New York onion fields. Environmental Entomology 35: 1264-1271. DOI: 10.1093/ee/35.5.1264.

Ogada P.A., Poehling H.M. 2015. Sex-specific influences of Frankliniella occidentalis (western flower thrips) in the transmission of Tomato spotted wilt virus (Tospovirus). Journal of Plant Diseases and Protection 122(5-6): 264-274. DOI: 10.1007/bf03356562.

Sogo K., Miura K., Aizawa M., Watanabe T., Stouthamer R. 2015. Genetic structure in relation to reproduction mode in Thrips tabaci (Insecta: Thysanoptera). Applied Entomology and Zoology 50: 73-77. DOI: 10.1007/s13355-014-0306-7.

Speyer E.R., Parr W.J. 1941. The external structure of some thysanopterous larvae. Transactions of the Royal Entomological Society of London 91: 559635. DOI: 10.1111/j.1365-2311.1941.tb01040.x.

Strauss S.Y., Karban R. 1994. The significance of outcrossing in an intimate plant-herbivore relationship. II. Does outcrossing pose a problem for thrips adapted to the host-plant clone? Evolution 48: 465476. DOI: $10.2307 / 2410105$.

Toda S., Murai T. 2007. Phylogenetic analysis based on mitochondrial COI gene sequences in Thrips tabaci Lindeman (Thysanoptera: Thripidae) in relation to reproductive forms and geographic distribution. Applied Entomology and Zoology 42: 309-316. DOI: 10.1303/aez.2007.309.

Vierbergen G.B., Kucharczyk H., Kirk W.D.J. 2010. A key to the second instar larvae of the Thripidae of the Western Palaearctic region (Thysanoptera). Tijdschrift voor Entomologie 153: 99-160. DOI: 10.1163/22119434-900000294. 